\title{
SOLUTIONS OF NONLINEAR BOUNDARY VALUE PROBLEM WITH APPLICATIONS TO BIOMASS THERMAL CONVERSION
}

\author{
Armands Gritsans ${ }^{1}$, Andrei Kolyshkin ${ }^{2}$, Diana Ogorelova ${ }^{1}$, Felix Sadyrbaev ${ }^{1}$, \\ Inna Samuilik ${ }^{1,2}$, Inara Yermachenko ${ }^{1}$ \\ ${ }^{1}$ Daugavpils University, Latvia; ${ }^{2}$ Riga Technical University, Latvia \\ armands.gricans@du.lv, andrejs.koliskins@ rtu.lv, diana.ogorelova@inbox.lv, felix@latnet.lv, \\ inna.samuilika@rtu.lv, inara.jermacenko@du.lv
}

\begin{abstract}
Bioenergy is one of the widespread renewable energy sources. Energy from biomass thermal conversion can reduce greenhouse emissions and have a positive effect on climate change. Biomass conversion is generally carried out in reactors of cylindrical shape. From a modelling point of view many factors have to be taken into account in order to optimize thermal efficiency of the conversion process. One of the important methods for the analysis of complex fluid flows is hydrodynamic stability theory. Base flow solution in classical hydrodynamic stability problems is usually found as a simple analytical solution of the equations of motion. Biomass conversion problems lead to nonlinear boundary value problems, which can be either solved numerically or analyzed using the bifurcation theory. In the present paper we analyze a mathematical model of heat transfer in the presence of nonlinear heat sources. This model includes the study of positive solutions to a nonlinear boundary value problem with certain boundary conditions. The equations in a problem contain several parameters, which essentially affect the behaviour and the number of solutions. Bifurcation analysis of the problem, conducted with respect to the parameters, allows obtaining somewhat precise results on the number of positive solutions. Generally, two, one and zero positive solutions are possible, depending on the values of the parameters. The obtained solutions represent base flow for the hydrodynamic stability problem, which can be solved with the objective to identify the factors affecting the conversion process.
\end{abstract}

Keywords: biomass thermal conversion; hydrodynamic stability; nonlinear boundary value problem; bifurcation analysis.

\section{Introduction}

Much attention is devoted nowadays to biomass thermal conversion with the objective to obtain more efficient and „green” energy sources. Energy conversion technologies are currently developed in many countries as a step towards bio-based economy, see [1-3]. Conversion process is a complex multiphysics problem, where combustion, heat transfer and fluid motion are coupled, see [4;5]. There are three major methods for investigation of complex physical processes in nature and engineering: experimental studies, numerical modeling, and stability analysis. The approach based on linear stability analysis is used in the present paper.

Equations of thermal convection under the Boussinesq approximation for a viscous incompressible fluid (in dimensionless form) are used to describe biomass thermal conversion:

$$
\begin{aligned}
\frac{\partial \vec{v}}{\partial t}+G r(\vec{v} \cdot \nabla) \vec{v} & =-\nabla p+\Delta \vec{v}+T \vec{k}, \\
\frac{\partial T}{\partial t}+G r \vec{v} \cdot \nabla T & =\frac{1}{\operatorname{Pr}} \Delta T+\frac{F}{\operatorname{Pr}} e^{T}, \\
\nabla \cdot \vec{v} & =0,
\end{aligned}
$$

where $\vec{v}$ - velocity vector;

$T$ - temperature;

$p$ - pressure;

$\vec{k}=(0,0,1)-$ unit vector in the vertical direction.

The flow is characterized by three parameters: the Grashof number $G r$, the Prandtl number $P r$, and the Frank-Kamenetskii parameter $F$. The flow is generated by internal heat sources that are distributed within the fluid due to chemical reactions in accordance with the Arrhenius law. The density of heat sources is a nonlinear function of the temperature; see the second term on the right-hand side of (2). Frank-Kamenetskii transformation, see [6], is used to transform the nonlinear term. 
In practice, thermal conversion of biomass takes place in cylindrical containers, see $[4 ; 5]$. Consider flow in a tall vertical annulus of inner and outer radii $R_{1}$ and $R_{2}$, respectively. The walls of the annulus are maintained at equal temperatures.

The next step in a linear stability analysis is to define the base flow (usually the flow of a simple structure satisfying the equations of motion). We assume that the base flow has the form

$$
\vec{v}_{0}=\left(0,0, v_{0}(r)\right), \quad T=T_{0}(r), \quad p=p_{01}(r)+p_{02}(z),
$$

where $(r, \theta, z)$ - system of cylindrical polar coordinates with the origin at the axes of the cylinders.

Substituting (4) into (1)-(3), we obtain the boundary value problem

$$
\begin{gathered}
v_{0}^{\prime \prime}+\frac{v_{0}^{\prime}}{r}+T_{0}=C, \\
T_{0}^{\prime \prime}+\frac{T_{0}^{\prime}}{r}+F e^{T_{0}}=0,
\end{gathered}
$$

where $C=-d p_{02} / d z-$ constant pressure gradient in the vertical direction.

The annulus is assumed to be closed, so that the total fluid flux through the cross-section is equal to zero:

$$
\int_{R}^{1} r v_{0}(r) d r=0
$$

where $\quad R=R_{1} / R_{2}$.

The boundary conditions are

$$
\begin{aligned}
& T_{0}(1)=T_{0}(R)=0, \\
& v_{0}(1)=v_{0}(R)=0 .
\end{aligned}
$$

It follows from (5)-(9) that the problem for the function $T_{0}(R)$ can be solved separately. In addition, nonlinearity in explicit form appears only in (6). Thus, the structure of the base flow is determined by the properties of the boundary value problem (6), (8). In the sequel we use bifurcation analysis to determine the structure and number of solutions of (6), (8).

\section{Bifurcation analysis}

To treat the problem (6), (8), let us consider first the auxiliary initial value problem

$$
T_{0}^{\prime \prime}(r)+\frac{T_{0}^{\prime}(r)}{r}+F e^{T_{0}(r)}=0, \quad T_{0}(1)=0, \quad T_{0}^{\prime}(1)=-\beta<0 .
$$

Acting like in [6], by the change of variables

$$
r=e^{-t}, \quad T_{0}(r)=w(t)+2 t,
$$

the problem (10) reduces to the initial value problem

$$
w^{\prime \prime}(t)+F e^{w(t)}=0, \quad w(0)=0, \quad w^{\prime}(0)=\beta-2 .
$$

Integrating the equation in (12), we obtain

$$
\pm \int_{w(0)}^{w(t)} \frac{d z}{\sqrt{(\beta-2)^{2}+2 F-2 F e^{z}}}=t .
$$

Taking into account [7] and the initial conditions in (12), we have 


$$
w(t)=\ln \left(\frac{4\left[(\beta-2)^{2}+2 F\right] e^{t \sqrt{(\beta-2)^{2}+2 F}}}{\left[\beta-2+\sqrt{(\beta-2)^{2}+2 F}+\left(\sqrt{(\beta-2)^{2}+2 F}-\beta+2\right) e^{t \sqrt{(\beta-2)^{2}+2 F}}\right]^{2}} .\right.
$$

It follows from (11) and (13) that the function

$$
T_{0}(r)=\ln \left(\frac{4\left[(\beta-2)^{2}+2 F\right] r^{\sqrt{(\beta-2)^{2}+2 F}-2}}{\left[2-\beta+\sqrt{(\beta-2)^{2}+2 F}+\left(\sqrt{(\beta-2)^{2}+2 F}+\beta-2\right) r^{\sqrt{(\beta-2)^{2}+2 F}}\right]^{2}}\right)
$$

solves the problem (10).

Suppose that $R$ is a fixed number in the interval $(0,1)$. If $T_{0}(r)$ is given by (14), then the equation $T_{0}(R)=0$ defines the bifurcation curve $\Gamma_{R}$ in the $(F, \beta)$-plane, which determines all positive solutions of the boundary value problem (6), (8). By calculations, $T_{0}(R)=0$, if and only if $g_{R}(F, \beta)=0$, where

$$
\begin{aligned}
g_{R}(F, \beta) & =4\left[(\beta-2)^{2}+2 F\right] R^{\sqrt{(\beta-2)^{2}+2 F}-2}- \\
- & {\left[\sqrt{(\beta-2)^{2}+2 F}-\beta+2+\left(\sqrt{(\beta-2)^{2}+2 F}+\beta-2\right) R^{\sqrt{(\beta-2)^{2}+2 F}}\right]^{2} . }
\end{aligned}
$$

Thereby,

$$
\Gamma_{R}=\left\{(F, \beta) \in \square^{2}: F>0, \beta>0, g_{R}(F, \beta)=0\right\} .
$$

Numerically analyzing the bifurcation curve $\Gamma_{R}$, we conclude that there exists a positive number $F^{*}$ such that the boundary value problem (6), (8) has

1. exactly two positive solutions if $F \in\left(0, F^{*}\right)$,

2. a unique positive solution if $F=F^{*}$,

3. no positive solutions if $F>F^{*}$.

Notice that these conclusions are consistent with the results obtained in [8;9] and they are valid for every aspect ratio $1 / R$. Item c) has a clear physical meaning: if the Frank-Kamenetskii parameter $F$ exceeds a certain value (that is, if the heat release as a result of the reaction is large), there is no steady solution of (6), (8). This situation is referred to as the thermal explosion in the literature, see [10].

\section{Numerical results and discussion}

In Fig. 1, the bifurcation curve $\Gamma_{R}$ in the $(F, \beta)$-plane is depicted for $R=0.3$. The vertical straight line $F=3$ and the curve $\Gamma_{R}$ intersect at the points $\left(F, \beta_{1}\right)=(3,1.0792)$ (in green) and $\left(F, \beta_{2}\right)=(3,9.9598)$ (in blue). Both points determine the positive solutions of the boundary value problem (6), (8) for $F=3$; see the green and blue graphs in Fig. 2. The bifurcation curve $\Gamma_{R}$ has a unique turning point $\left(F^{*}, \beta^{*}\right)=(6.9208,4.6566)$ (in brown). The last point determines a unique positive solution of $(6),(8)$ for $F=F^{*}$; see the brown graph in Fig. 2. It is seen from Fig. 2 that the green curve should be interpreted as the base flow temperature distribution in linear stability analysis (physically realizable distribution is the one with smaller temperature values). Thus, the analysis of different solutions helps select the base flow profile for linear stability.

The graphs of the two solutions for the base flow temperature distribution in an annulus with $R=0.3$ and $F=3$ are shown in Fig. 3; we plot the graphs of

$$
u(x, y)=T_{0}\left(\sqrt{x^{2}+y^{2}}\right)
$$

for $\beta_{1}=1.0792$ (green curves) and $\beta_{2}=9.9598$ (blue curves), where $T_{0}$ is defined by (14). The physically realizable case is represented by green curves. 

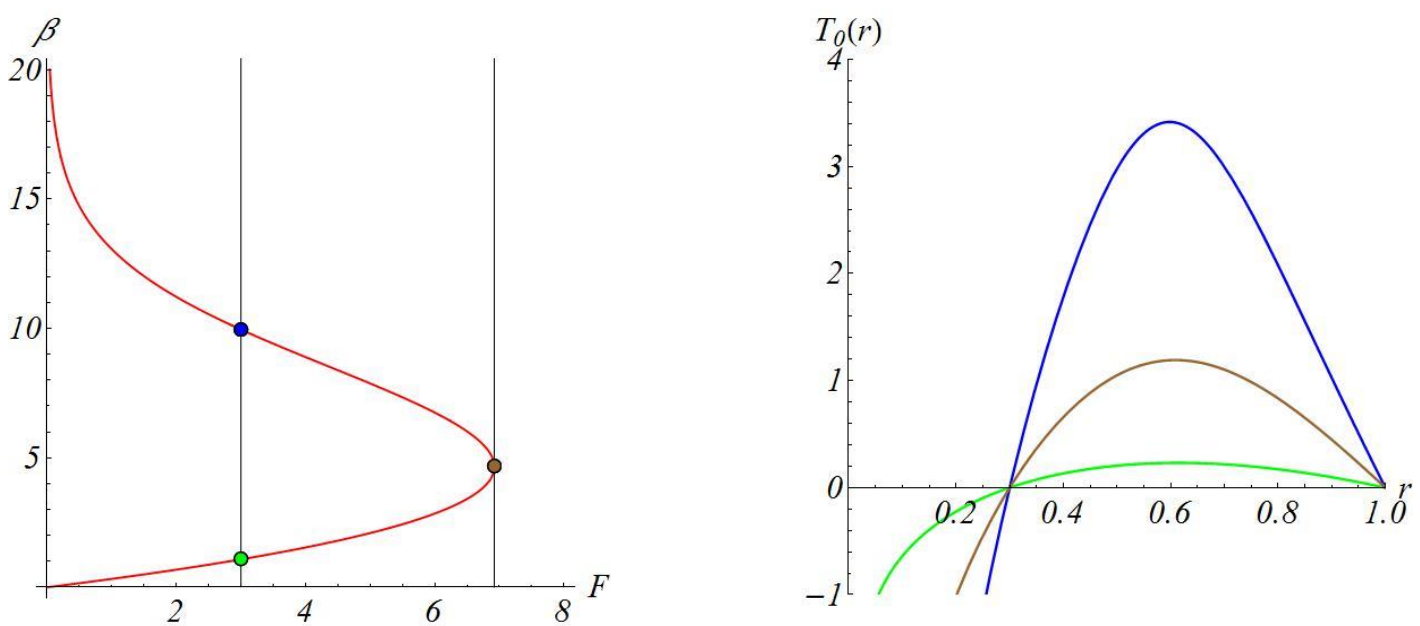

Fig. 1. Bifurcation curve $\Gamma_{R}$ if $\boldsymbol{R}=\mathbf{0 . 3}$

Fig. 2. Three positive solutions of $(6),(8)$ if $R=0.3$

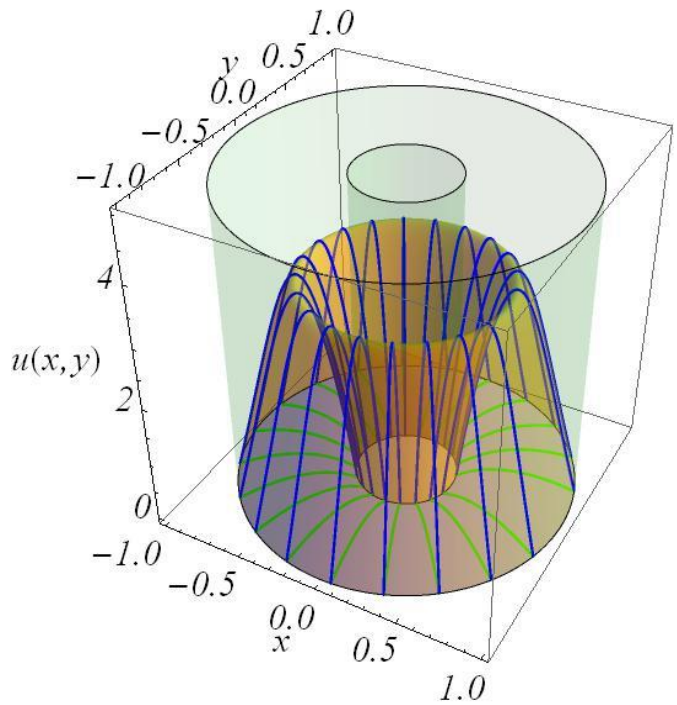

Fig. 3. Two solutions of the base flow temperature distribution

Let us investigate the evolution of bifurcation curves $\Gamma_{R}$, if the positive parameter $R$ in (15) changes from zero to one. For a fixed positive $R$ in the interval $(0,1)$, a point $\left(F^{*}, \beta^{*}\right) \in \Gamma_{R}$ is a turning point of $\Gamma_{R}$, if the tangent line

$$
\frac{\partial g_{R}}{\partial F}\left(F^{*}, \beta^{*}\right)\left(F-F^{*}\right)+\frac{\partial g_{R}}{\partial \beta}\left(F^{*}, \beta^{*}\right)\left(\beta-\beta^{*}\right)=0
$$

of $\Gamma_{R}$ at the point $\left(F^{*}, \beta^{*}\right)$ is vertical in the $(F, \beta)$-plane, that is,

$$
\frac{\partial g_{R}}{\partial \beta}\left(F^{*}, \beta^{*}\right)=0 .
$$

Hence, the turning points of $\Gamma_{R}$ can be determined from the following system:

$$
g_{R}(F, \beta)=0, \frac{\partial g_{R}}{\partial \beta}(F, \beta)=0 .
$$

We can approximate the data $\left\{F^{*}, \beta^{*}\right\}$ from Table 1 by the function $\beta=\phi(F)$, where

$$
\phi(F)=54.7794 \sqrt[5]{F}-70.8056 \sqrt[4]{F}+22.7043 \sqrt[3]{F}+0.759267 \sqrt{F}+0.0021133 F-6.43759 .
$$

Then, $\max \left|\phi\left(F^{*}\right)-\beta^{*}\right|<10^{-3}$ for all the pairs $\left\{F^{*}, \beta^{*}\right\}$ from Table 1 . Similarly, we can approximate the data $\left\{R, F^{*}\right\}$ from Table 1 by the function $F=\psi(R)$, where 


$$
\begin{gathered}
\psi(R)=2.87508+9.25825 R+8.08997 R^{2}+5.15669 R^{3}+48.3825 R^{4}+172.69 R^{8}+ \\
+668.605 R^{16}+2544.45 R^{32}+10531.6 R^{64} .
\end{gathered}
$$

Then, $\max |\psi(R)-R|<10^{-2}$ for all the pairs $\left\{R, F^{*}\right\}$ from Table 1 .

Numerical values of turning points $\left(F^{*}, \boldsymbol{\beta}^{*}\right)$ for some $\boldsymbol{R}$

Table 1

\begin{tabular}{|c|c|c|c|c|c|}
\hline $\boldsymbol{R}$ & $\boldsymbol{F}^{*}$ & $\boldsymbol{\beta}^{*}$ & $\boldsymbol{R}$ & $\boldsymbol{F}^{*}$ & $\boldsymbol{\beta}^{*}$ \\
\hline 0.100 & 3.89151 & 3.21747 & 0.750 & 56.0988 & 15.0805 \\
\hline 0.200 & 5.17081 & 3.8792 & 0.775 & 69.2902 & 16.863 \\
\hline 0.300 & 6.92089 & 4.65662 & 0.800 & 87.7304 & 19.0898 \\
\hline 0.400 & 9.55557 & 5.65338 & 0.825 & 114.625 & 21.9514 \\
\hline 0.500 & 13.8823 & 7.0206 & 0.850 & 156.061 & 25.7652 \\
\hline 0.600 & 21.8124 & 9.04783 & 0.875 & 224.779 & 31.1027 \\
\hline 0.700 & 38.9122 & 12.4038 & 0.900 & 351.28 & 39.1067 \\
\hline 0.725 & 46.3376 & 13.6211 & - & - & - \\
\hline
\end{tabular}

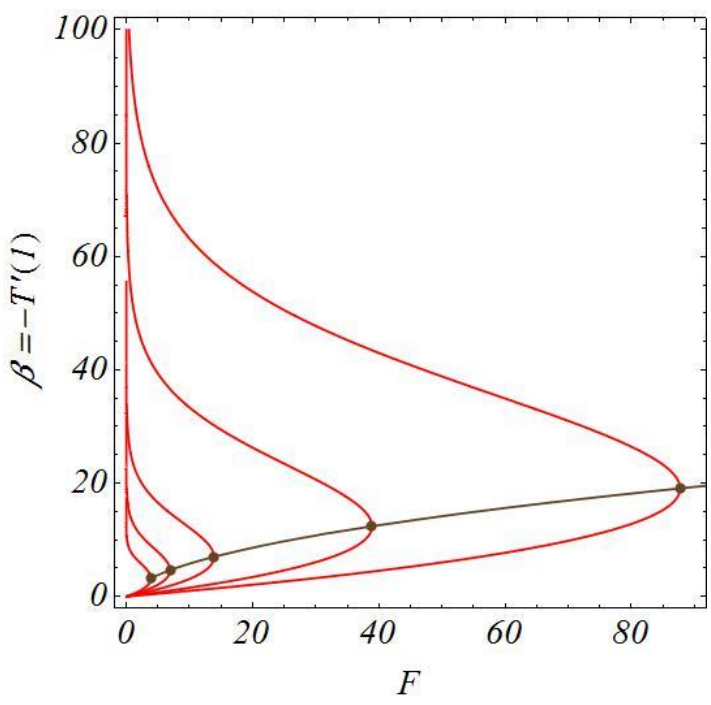

Fig. 4. Evolution of bifurcation curves

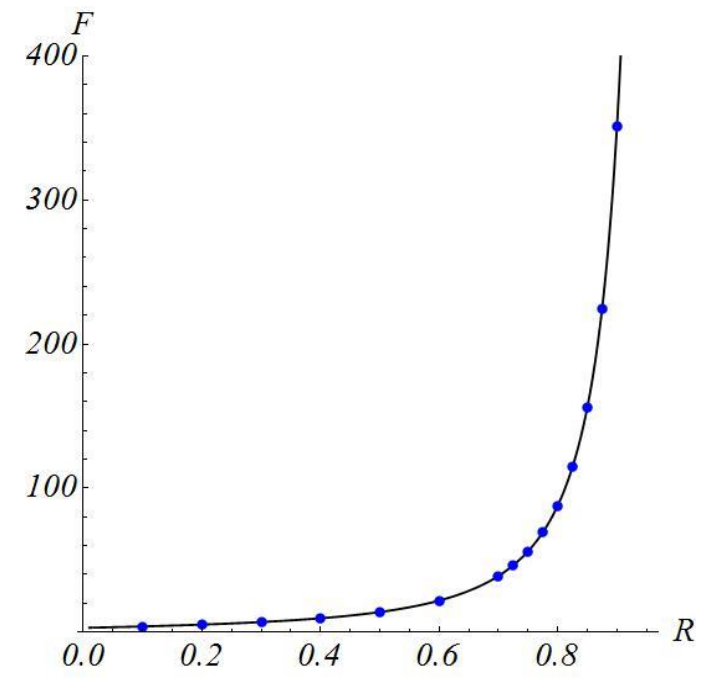

Fig. 5. Threshold value of $\boldsymbol{F}$ versus $\boldsymbol{R}$

In Fig. 4, the evolution of bifurcation curves $\Gamma_{R}$ (in red) is shown, when $R=0.1,0.3,0.5,0.7,0.8$ (from left to right); in the same figure, the graph of the function $\beta=\phi(F)$ passing through the corresponding turning points is displayed (in brown). In Fig. 5, the pairs $\left(R, F^{*}\right)$ (in blue) from Table 1 and the graph (in black) of the function $F=\psi(R)$ are shown. The graph in Fig. 5 shows for which values of the radius ratio $R$ and the Frank-Kamenetskii parameter $F$ there exist a steady solution of (6), (8); in the same figure, if a point $(R, F)$ is located below the graph, then there exists a physically realizable solution to (6), (8) and if a point $(R, F)$ is located above the graph, then the problem (6), (8) has no solutions and thermal explosion takes place.

Let us consider the elliptic boundary value problem

$$
\Delta T+F e^{T}=0,\left.\quad T\right|_{\partial \Omega}=0,
$$

where $\partial \Omega$ is the boundary of a domain $\Omega$. It is shown in $[10 ; 11]$ that closed form solutions exist for the problem (16) assuming planar geometry (region between two infinitely long parallel vertical planes) and cylindrical geometry (region inside an infinitely tall cylinder of a circular cross-section). Numerical solution is found in $[10 ; 11]$ for the case of spherical geometry (region inside a ball). In the present paper the problem (16) or (6), (8) is solved in a region between two concentric infinitely long cylinders using methods of the bifurcation theory. In addition to the Frank-Kamenetskii parameter $F$, there is also another parameter $R$ (the radius ratio of the cylinders). The number of steady solutions and their structure for the problem (6), (8) as a function of $F$ and $R$ is completely described in this paper. 


\section{Conclusions}

1. The nonlinear boundary value problem for the base flow in a tall vertical annulus generated by internal heat sources is formulated in the paper.

2. Bifurcation analysis of the nonlinear boundary value problem for the temperature distribution is performed for different values of the parameters of the problem: the radius ratio and the FrankKamenetskii parameter.

3. It is shown that there are two, one, or no positive solutions of the boundary value problem for the temperature distribution depending on the values of the parameters $F$ and $R$

4. In case of two positive solutions bifurcation analysis defines a physically realizable temperature profile, which can be further used in the stability analysis of such flows.

5. The results of the present study can be used to define the base flow for hydrodynamic stability analysis of the biomass thermal conversion problem in cylindrical containers with the objective to identify factors stimulating mixing and more efficient energy conversion. Comprehensive analysis of instabilities can be used in the design of bioreactors.

\section{Acknowledgements}

This research is funded by the Latvian Council of Science, project "Analysis of complex dynamical systems in fluid mechanics and heat transfer", project No. 1zp-2020/1-0076.

\section{References}

[1] Lewandowski W.M., Ryms M., Kosakowski W. Thermal biomass conversion: A review. Processes, vol. 8, 2020, 516. [online] [21.03.2021] Available at: https://www.mdpi.com/2227-9717/8/5/516.

[2] Yamakawa C.K., Qin F., Mussatto S.I. Advances and opportunities in biomass conversion technologies and biorefineries for the development of bio-based economy. Biomass and bioenergy, vol. 119, 2018, pp. 54-60.

[3] Kataki R., Pandley A., Khanal S.K., Pant D. (eds.). Current developments in biotechnology and bioengineering. Sustainable bioresources for emerging bioeconomy, Elsevier, 2020, 536 p.

[4] Barmina I., Purmalis M., Valdmanis R, Zake M. Electrodynamic control of the combustion characteristics and heat energy production. Combustion Science and Technology, vol. 188, 2016, pp. 190-206.

[5] Kalis H., Marinaki M., Strautins U., Zake M. On numerical simulation of electromagnetic field effects in the combustion process. Mathematical Modeling and Analysis, vol. 23, 2018, pp. 327343. [online] [21.03.2021] Available at: https://journals.vgtu.lt/index.php/MMA/article/view/1434/1143.

[6] Bebernes J., Eberly D. Mathematical problems from combustion theory. New York: SpringerVerlag, 1989. $177 \mathrm{p}$.

[7] Prudnikov A.P., Brychkov Yu.A., Marichev O.I. Integrals and series. Volume 1. Elementary functions. Second revised edition. Moscow: Fiziko-Matematicheskaya Literatura, 2003. 632 p. (In Russian).

[8] Lin S.S. On non-radially symmetric bifurcation in the annulus. Journal of Differential Equations, vol. 80, 1989, pp. 251-279.

[9] Lin S.S. Positive radial solutions and nonradial bifurcation for semilinear elliptic equations in annular domains. Journal of Differential Equations, vol. 86, 1990, pp. 367-391.

[10]Zeldovich Ya.B., Barenblatt G.I., Librovich V.B., Makhviladze G.M. Mathematical theory of combustion and explosions. New York: Consultants Bureau [Plenum], 1985. 597 p.

[11] Frank-Kamenetskii D.A. Diffusion and heat exchange in chemical kinetics. Princeton: Princeton University Press, 2015, 384 p. 